\title{
ArcheoSciences
}

Revue d'archéométrie

$30 \mid 2006$

Varia

\section{Minéralogie des silicates de calcium présents dans des mortiers anciens à Tournai}

Mineralogy of the calcium-silicate phases present in ancient mortars from Tournai

Gilles Mertens, Jan Elsen, Dominique Laduron et Raymond Brulet

\section{(2) OpenEdition}

Journals

Édition électronique

URL : https://journals.openedition.org/archeosciences/150

DOI : 10.4000/archeosciences. 150

ISBN : 978-2-7535-1595-6

ISSN : 2104-3728

Éditeur

Presses universitaires de Rennes

Édition imprimée

Date de publication : 31 décembre 2006

Pagination : 61-65

ISBN : 978-2-7535-0456-1

ISSN : $1960-1360$

Référence électronique

Gilles Mertens, Jan Elsen, Dominique Laduron et Raymond Brulet, « Minéralogie des silicates de calcium présents dans des mortiers anciens à Tournai », ArcheoSciences [En ligne], 30 | 2006,

document 5, mis en ligne le 31 décembre 2008, consulté le 01 février 2022. URL : http://

journals.openedition.org/archeosciences/150; DOI : https://doi.org/10.4000/archeosciences.150

Article L.111-1 du Code de la propriété intellectuelle. 


\title{
Minéralogie des silicates de calcium présents dans des mortiers anciens à Tournai
}

\author{
Gilles Mertens*, Jan Elsen**, Dominique Laduron*** et Raymond Brulet****
}

Résumé : Cette étude est destinée à clarifier l'évolution au niveau de la composition et des techniques de fabrication des mortiers à chaux utilisés dans les structures archéologiques découvertes sous le niveau de sol actuel de la cathédrale de Tournai (Belgique) qui remonte au XII ${ }^{e}$ siècle. La recherche s'inscrit dans un projet interdisciplinaire plus large qui vise à étudier la transition des villes et des territoires ruraux Romains à PostRomain et leur transformation jusqu’au Moyen Âge (Brulet et Verslype, 2001). Les résultats présentés, concernent la minéralogie des mortiers et indiquent que celle-ci peut varier selon l'hydraulicité du liant, qui à son tour est mis en relation avec les conditions de cuisson du calcaire dans les fours à chaux anciens. Cette étude est donc particulièrement intéressante lorsqu'il s’agit de comprendre et de clarifier la connaissance empirique possible de l'interdépendance de la température de cuisson et de l'hydraulicité de la chaux utilisée à Tournai. Il est en effet possible que pour certaines applications ou durant certaines époques de l'histoire cette connaissance fût exploitée.

Abstract: The aim of this study is to clarify the evolution of the composition and techniques of ancient lime mortars used in the archaeological structures which can be found beneath the present-day floor level of the $12^{\text {th }}$ century cathedral of Tournai (Belgium). The research is part of a broader interdisciplinary project considering the transition of ancient cities and their rural territories from Roman to late Roman society and the transformation to the early Middle Ages (Brulet \& Verslype, 2001). The results presented, concern the mineralogy of the mortars which is shown to be related to their hydraulicity, which is on its turn related to the burning conditions of the limestone in the ancient lime kilns. This study is of a particular interest when it comes to understand and clarify the possible empirical knowledge of the interdependance of the burning temperature and the hydraulicity of the lime used at Tournai. It is indeed possible that for some applications or during some time in history, this knowledge was exploited.

Mots clés : Cuisson de la chaux, hydraulicité, minéralogie, mortiers à chaux anciens, silicates de calcium.

Key words: Ancient lime mortars, calcium silicates, hydraulicity, lime burning, mineralogy.

\section{INTRODUCTION}

Depuis 1986, d'importantes excavations archéologiques sont menées autour et à l'intérieur de la cathédrale NotreDame de Tournai (Belgique), qui a récemment été ajoutée à la liste du Patrimoine Mondial de l'Unesco (Fig. 1). Les excavations sont principalement liées aux travaux de stabi- lisation des fondations de la cathédrale. Depuis quelques années, les fouilles sont menées dans le cadre d'un projet dirigé par l'Université Catholique de Louvain et financé par la Direction de l'Archéologie en Région wallonne. Des structures découvertes, les plus anciennes datent de l'époque romaine. Elles sont recouvertes par les restes d'une basilique Paléochrétienne dont une partie des murs et des

\footnotetext{
* Étudiant de doctorat, Katholieke Universiteit Leuven, Afdeling geologie, Celestijnenlaan 200E, 3001 Heverlee, Belgique. (Gilles.Mertens@geo.kuleuven. ac.be)

** Katholieke Universiteit Leuven, Afdeling geologie, Celestijnenlaan 200E, 3001 Heverlee, Belgique. (Jan.Elsen@geo.kuleuven.ac.be)

*** Université catholique de Louvain, Unité de Géologie, Place L. Pasteur 3, 1348 Louvain-la-Neuve, Belgique. (Laduron@geol.ucl.ac.be)

**** Université catholique de Louvain, Centre de recherches d'archéologie nationale, Place Blaise Pascal 1, 1348 Louvain-la-Neuve, Belgique. (Brulet@ arke.ucl.ac.be)
} 


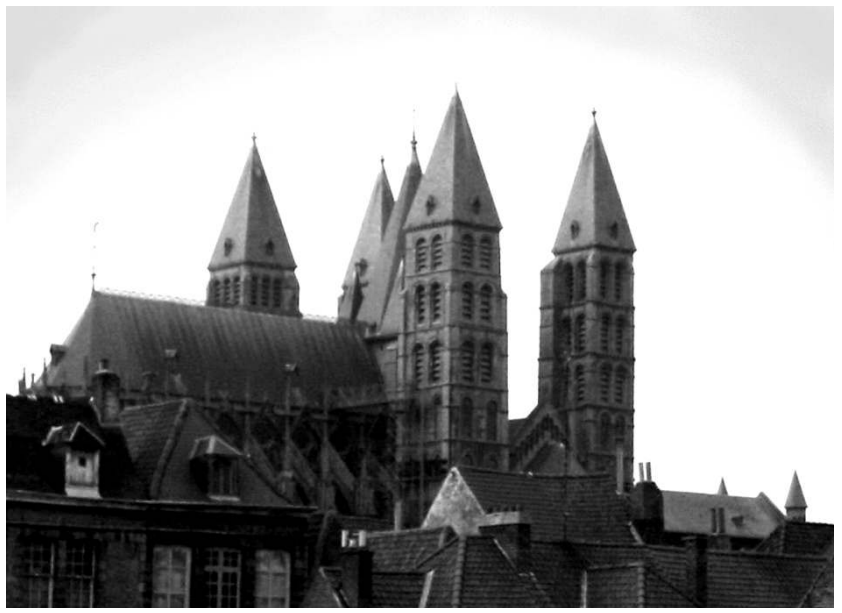

Figure 1 : Cathédrale romane Notre-Dame de Tournai (Belgique). Figure 1: Romanesque cathedral Notre-Dame of Tournai (Belgium).

locaux annexes sont préservés. D’une époque plus récente, on doit mentionner les restes d'églises du haut Moyen Âge, notamment carolingienne, avec un baptistère. Vers l'an Mil, l'église fut entièrement reconstruite. Le plan de cette dernière église est très similaire à celui de la cathédrale romane actuelle.

D'un point de vue géologique, le calcaire d'âge tournaisien affleurant dans la vallée de l'Escaut, entre Tournai et Antoing, a été de tout temps l'objet d'une importante extraction. Il y a de nombreuses preuves de son utilisation dans le Tournaisis à l'époque gallo-romaine, tant comme pierre de taille que comme pierre à chaux (Camerman, 1944; Mortelmans, 1969). Une caractéristique générale du calcaire est son imprégnation de silice microcristalline qui a conduit localement à des concrétions sous forme de cherts. Par ailleurs, les teneurs en argiles du calcaire sont variables (Gaillard et al., 1999).

\section{1. ÉNONCÉ DU PROBLÈME}

Jusqu'à présent vingt échantillons de mortier ont été prélevés sur les différentes constructions découvertes. Une partie de chacun de ces échantillons a été utilisée pour la préparation d'une lame mince, l'autre partie a été utilisée pour l'analyse minéralogique et chimique. Pour déterminer l'hydraulicité du liant, la fraction $<65 \mu \mathrm{m}$, séparée après un léger broyage du mortier dans un mortier en agate, a été analysée chimiquement selon la procédure proposée par RILEM (Middendorf et al., 2000). Lors de cette procédure, cette fraction enrichie en liant est dissoute dans l'acide chlorhydrique et analysée par AAS (spectrométrie d'absorption atomique). Les résultats montrent que les liants de la plu- part des mortiers $(16 / 20)$ ont un caractère non-hydraulique (Mertens et al., 2005; Elsen et al., 2004) avec un indice de cimentation (I.C.) inférieur à 0,3 (Boynton, 1966).

$$
\text { I.C. }=\frac{1,1 . \% \mathrm{Al}_{2} \mathrm{O}_{3}+0,7 . \% \mathrm{Fe}_{2} \mathrm{O}_{3}+2,8 \% \mathrm{SiO}_{2}}{\% \mathrm{CaO}+1,4 . \% \mathrm{MgO}}
$$

Par ailleurs, trois mortiers peuvent être classés comme " éminemment hydrauliques ", c'est-à-dire ayant un indice de cimentation compris entre 0,7 et 1,1 .

La chaux de Tournai a également été utilisée dans la construction ou la restauration d'autres bâtiments en dehors du bassin de l'Escaut, tels que l'église Saint-Michel à Leuven. Les mortiers utilisés lors de la restauration de cette église baroque au XIX $x^{e}$ siècle (1853-1880) ont également un caractère "éminemment hydraulique " (Callebaut et al., 2001).

Plusieurs hypothèses quant à cette différence en hydraulicité des liants peuvent être avancées. La première est de considérer différentes sources de calcaire pour la production de la chaux. Cette explication est cependant peu plausible vue l'abondance locale de calcaire. La présence sporadique de morceaux de calcaire non-cuits dans certains mortiers non-hydrauliques indiquent clairement une provenance locale de ceux-ci. On y observe en effet, des crinoïdes, brachiopodes et bryozoaires qui font partie de la faune typique des calcaires biomicritiques du Tournaisien.

Une deuxième hypothèse reviendrait à expliquer la différence en hydraulicité par une variation dans la composition du calcaire local. Même si cette différence existe, le calcaire est caractérisé par une teneur élevée en silice (Camerman, 1944), variant de $7,5 \%$ à $24 \%$ (Hennebert \& Doremus, 1997), une concentration que l'on ne retrouve pas dans tous les mortiers.

Dans une troisième hypothèse, on pourrait considerer l'agrégat comme étant à l'origine de la différence en hydraulicité. En effet, le tuileau peut provoquer une réaction pouzzolanique engendrant la formation de silicates et aluminates de calcium hydratés (Coutelas et al, 2004) qui serait à leur tour responsable du caractère hydraulique de certains mortiers. Cependant, les échantillons présentant un caractère éminemment hydraulique n'ont pas (1 échantillon) ou très peu de tuileau ( $<1 \%$ en volume pour les deux autres échantillons - établi par comptage des points) comme agrégat. L'effet pouzzolanique du tuileau n'intervient donc pas ou seulement partiellement dans le développement de l'hydraulicité pour les échantillons étudiés.

La dernière hypothèse, la plus probable, consiste à considérer la température de cuisson du calcaire comme étant à l'origine de la différence en hydraulicité des chaux produites. À une température plus élevée ou temps de cuisson plus 
long, certaines réactions pourraient se produire qui ne se produiraient pas à température plus basse ou temps de cuisson moins long. Selon la minéralogie ainsi formée, le taux de silice soluble dans les chaux, et par conséquent dans les mortiers, varierait. Pour vérifier cette hypothèse une étude de la minéralogie des mortiers a été réalisée.

\section{Méthodologie}

\subsection{Microscopie}

Létude microscopique des lames minces montre que les mortiers sont constitués d'un liant à base de chaux et d'un agrégat de tuileau ou de sable fin glauconieux, ou d'un mélange des deux. La taille moyenne du sable varie de 150 à $200 \mu \mathrm{m}$. La proportion de tuileau et la taille des fragments semble mieux contrôlée dans les mortiers Romains. Les proportions chaux/agrégat sont très variables, allant de 0,3 à 2,5 , et ne montrent à première vue pas de corrélation avec le type de structure dans lequel ils sont utilisés. Il semble cependant que les mortiers de l'époque gallo-romaine et paléo-chrétienne sont plus riches en liant (proportion de 1,6 à 2,5) par rapport aux mortiers préromans et romans (proportion de 0,3 à 2,0 ). Une caractéristique générale des mortiers est la présence de fragments de cherts qui ne proviennent pas de l'agrégat utilisé, mais qui semblent, vu leur taille, provenir du calcaire utilisé lors de la fabrication de la chaux (Fig. 2).

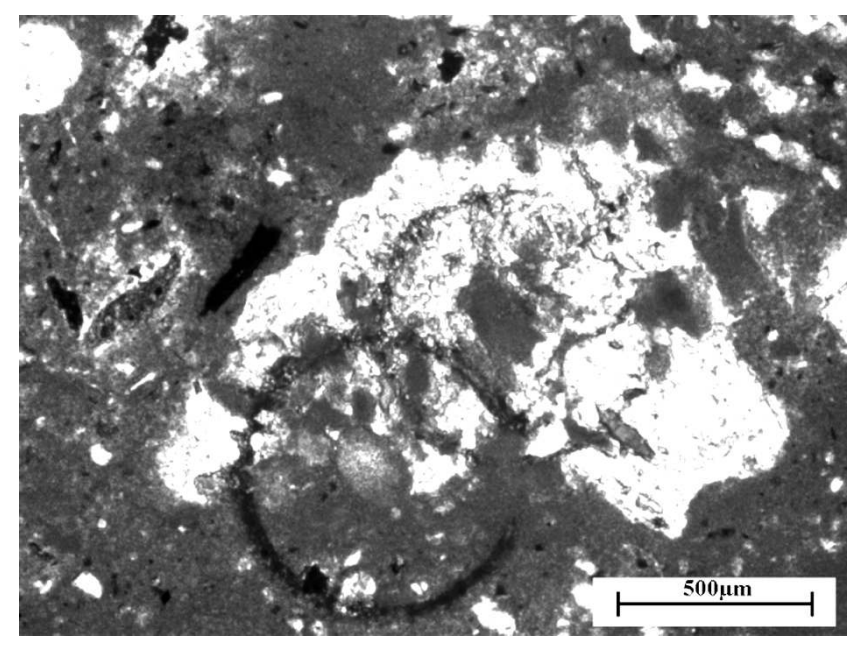

Figure 2 : Fragment de cherts en lame mince sous microscope à lumière transmise non polarisée. Le liant entourant le fragment est composé de $\mathrm{CaCO}_{3}$ pur (échantillon GR03 : mortier nonhydraulique).

Figure 2: Unpolarized transmitted light microscopic view of a flint fragment in a thin section. The binder surrounding the fragment is composed of pure $\mathrm{CaCO}_{3}$ (Sample GRO3 : non-hydraulic mortar).
La taille de ces fragments varie d'une centaine de micromètres jusqu'à plusieurs millimètres. En microscopie à lumière polarisée, on peut par ailleurs détecter certaines zones de taille similaire qui ont des teintes d'interférence différentes comparées aux cherts et qui sont uniquement présentes dans les mortiers hydrauliques. Ces fragments (Fig. 3) ont souvent un aspect amorphe avec parfois quelques cristaux euhédraux, le plus souvent orientés vers l'intérieur des cavités quand elles sont présentes.

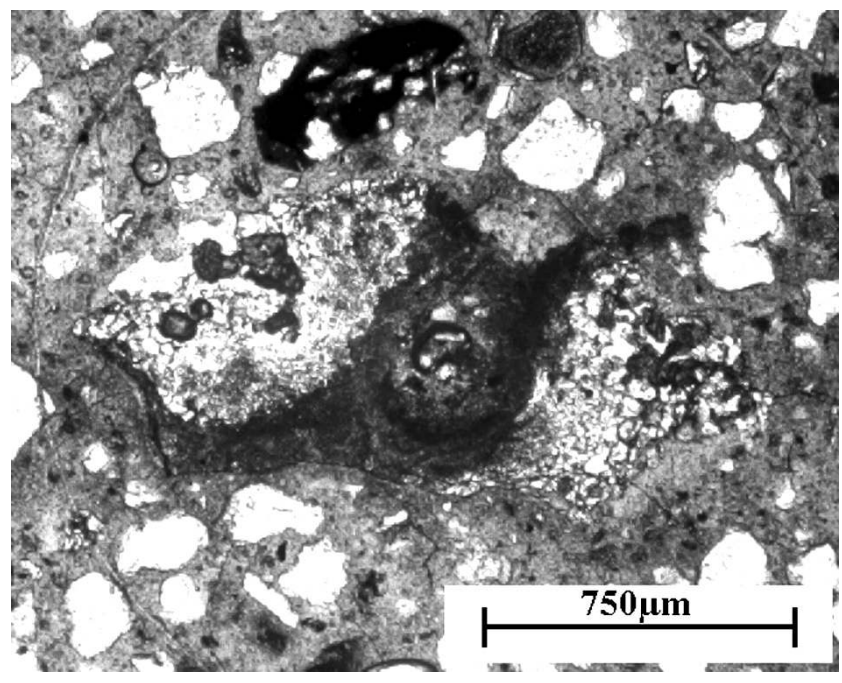

Figure 3: Agrégat polycristallin ressemblant aux fragments de cherts sous microscope à lumière transmise non polarisée (échantillon CA01 : mortier hydraulique).

Figure 3: Unpolarized transmitted light microscopic view of a polycrystalline aggregate resembling a flint fragment (sample CAO1 : hydraulic mortar).

\subsection{Diffraction des rayons $X$}

Les fractions $<65 \mu \mathrm{m}$ riches en liant ont également été examinées par diffraction des rayons $\mathrm{X}$. Ces analyses ont montré que les constituants cristallins principaux de cette fraction sont la calcite et le quartz, avec parfois quelques traces de feldspaths. La présence de calcite et l'absence de portlandite montrent que les liants sont entièrement carbonatés. Le quartz fait surtout partie de la fraction fine du sable de l'agrégat, mais il n'est pas exclu qu'une partie soit originaire du liant. En effet, une fraction importante de la silice libre dans les calcaires Tournaisiens se présente sous la forme de quartz (Philippo, 1992) qui est susceptible de rester, au moins partiellement, dans la chaux. Les feldspaths font également partie de la fraction fine de l'agrégat. Dans les spectres de diffraction des mortiers hydrauliques, on peut également discerner une zone où l'intensité du rayonnement devient plus intense sans prendre la forme d'un pic discret. 
Cette zone entre $29,5^{\circ}$ et $32^{\circ} 2 \theta(\mathrm{CuK \alpha})$, ce qui correspond à des distances inter-atomiques de 3,03 $\AA$ et 2,80 , est la zone où les silicates de calcium hydratés (phase C-S-H) peu cristallins montrent une diffraction (Ito et al., 1992). Il s'agit d'une phase à structure peu ordonnée qui se forme entre autre par hydratation des silicates de calcium plus courants $\left(\mathrm{Ca}_{2} \mathrm{SiO}_{4}\right.$ et $\left.\mathrm{Ca}_{3} \mathrm{SiO}_{5}\right)$ et présents dans les chaux hydrauliques et les ciments. Dans une tentative d'identifier les minéraux inconnus dans les mortiers hydrauliques mais repérés par microscopie, la partie non-soluble à l'HCl de la fraction $>65 \mu \mathrm{m}$ a également été analysée par diffraction des rayons $\mathrm{X}$. Les spectres révèlent cependant uniquement les minéraux appartenant à l'agrégat. Pour mieux cerner la zone d'intérêt, des analyses par microdiffraction des rayons $\mathrm{X}$ ont été effectuées sur certaines lames minces. Ces analyses ont montré qu'il s'agit de rankinite $\left(\mathrm{Ca}_{3} \mathrm{Si}_{2} \mathrm{O}_{7}\right)$ et de wollastonite $\left(\mathrm{CaSiO}_{3}\right)$, deux minéraux souvent associés aux roches carbonatées impures métamorphiques. Les spectres de diffraction ne permettent cependant pas de différencier les deux polymorphes de la wollastonite. La pseudowollastonite se forme à partir de $1125^{\circ} \mathrm{C}$, tandis que la parawollastonite est un polymorphe de basse température (Taylor, 1990).

\subsection{Microsonde}

La minéralogie des phases non-identifiées par microscopie a également été étudiée à la microsonde électronique. La phase avec une composition chimique de 51,43\% $( \pm 0,33)$ en $\mathrm{SiO}_{2}$ et de 48,02\% $( \pm 0,45)$ en $\mathrm{CaO}$ est interprétée comme étant la wollastonite. Elle n'apparaît pas isolément, mais est toujours associée à une phase C-S-H dont la composition varie entre $30-35 \% \mathrm{SiO}_{2}$ et 33-39\% CaO. Parfois, une troisième phase peut être identifiée. Sa composition avec 41,67\% $( \pm 0,03)$ de $\mathrm{SiO}_{2}$ et de $57,84 \%( \pm 0,99)$ en $\mathrm{CaO}$ montre qu'il s'agit de rankinite. D'autres phases à composition variable ne correspondent pas à des composés cristallins connus. Il s'agit probablement de phases amorphes. Le mode d'association de ces différentes phases ne semble pas suivre une logique constante. On relèvera que la phase C-S-H se situe le plus souvent en bordure des autres phases ou des cavités présentes. Parfois, la rankinite est présente en absence de la wollastonite, mais dans ce cas, elle est invariablement associée à une phase C-S-H (Fig. 4). Dans la plupart des mortiers hydrauliques, les pores dans le liant sont remplis de sels d'alumine hydratés comme l'hydrocalumite ou l'ettringite.

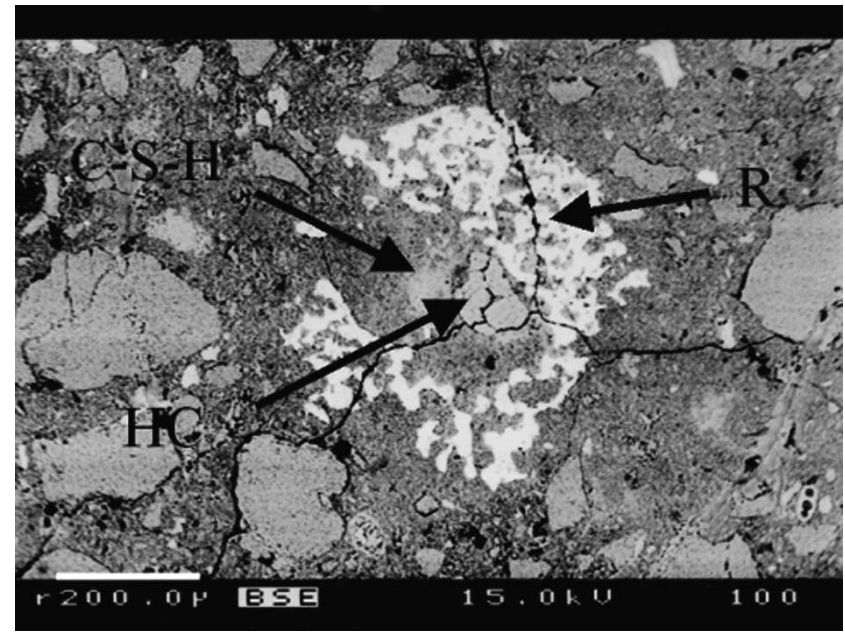

Figure 4 : Agrégat polycristallin semblable à celui de la figure 3 . Image en électrons rétrodiffusés de la rankinite ( $\mathrm{R}$ - phase claire) avec au centre un grain d'hydrocalumite $\left(\mathrm{HC}-\mathrm{Ca}_{2} \mathrm{Al}(\mathrm{OH})_{7} \cdot 3 \mathrm{H}_{2} \mathrm{O}\right)$ fracturé et une phase $\mathrm{C}-\mathrm{S}-\mathrm{H}$ immédiatement à gauche de ce dernier (phase gris clair). (Echantillon CA01 : mortier hydraulique). Figure 4: Poly-crystalline aggregate similar to the one in figure 3. Backscattered electron image of rankinite ( $R$ - light colored phase) with in the center a fractured grain of hydrocalumite (HC $\mathrm{Ca}_{2} \mathrm{Al}(\mathrm{OH})_{7} \cdot 3 \mathrm{H}_{2} \mathrm{O}$ ) and a $\mathrm{C}$-S-H phase immediately left of it (light gray phase). (Sample CAO1 : hydraulic mortar).

\section{INTERPRÉTATION ET CONCLUSIONS}

L'étude des mortiers anciens découverts dans la cathédrale de Tournai montre que deux types de liants existent. D'une part, on distingue des mortiers à chaux hydraulique et d'autre part des mortiers à chaux non-hydraulique. Une analyse minéralogique montre une différence considérable entre ces deux types de mortiers, dont la chaux est probablement issue du même calcaire siliceux, localement disponible. Cette différence consiste en la présence de wollastonite, de rankinite, de phases amorphes et d'une phase C-S-H, des minéraux qui n'ont pas été identifiés dans les mortiers non-hydrauliques. Des fragments de cherts sont présents dans les deux types de mortiers et semblent provenir du calcaire utilisé pour la production de la chaux. La différence en hydraulicité s'explique donc par une minéralogie distincte, qui est interprétée comme étant le résultat d'une température ou d'un temps de cuisson variable d'un même type de calcaire dans les fours à chaux. Les minéraux comme la wollastonite et la rankinite se forment en effet uniquement sous des conditions de températures plus élevées et/ou des temps de cuisson plus longs. Dans des conditions de production moins extrêmes, ces minéraux ne se forment pas dans la chaux. La phase C-S-H, qui est présente uniquement dans les mortiers hydrauliques, n'est 
pas une phase 'primaire', mais s'est probablement formée par hydratation de silicates de calcium comme le $\mathrm{Ca}_{2} \mathrm{SiO}_{5}$ ou le $\mathrm{Ca}_{3} \mathrm{SiO}_{6}$. Ces minéraux n'ont pas été identifiés et ont donc sans doute été entièrement hydratés. La phase C-S-H est à l'origine de la teneur élevée en $\mathrm{SiO}_{2}$ dans la fraction soluble à l'HCl. C'est cette teneur élevée en $\mathrm{SiO}_{2}$ qui mène à un indice de cimentation élevé, et donc à une classification comme mortier hydraulique. La teneur peu élevée en $\mathrm{SiO}_{2}$ soluble dans les mortiers non-hydrauliques est par contre liée à la faible concentration en C-S-H.

Cette étude montre que la connaissance de la minéralogie des mortiers peut apporter des informations considérables à l'étude des techniques de production de la chaux et des mortiers dans le passé. La minéralogie peut entre autres aider à déterminer les conditions de cuisson dans les fours à chaux.

Etablir un lien entre l'hydraulicité des mortiers et la position architecturale ou la période de fabrication des mortiers n'est pas évident vu le nombre limité d'échantillons. Les trois mortiers éminemment hydrauliques sont tous d'âge post-romain. Un d'entre eux (CA01) provient du baptistère, exigeant en effet un mortier imperméable, motivant sans doute le recours à une chaux hydraulique.

La recherche se poursuivra par des cuissons du calcaire de Tournai, prévues dans le but de mettre en évidence d'une manière expérimentale la minéralogie résultant de ces conditions de cuisson. D'autre part, l'étude d'un plus grand nombre d'échantillons de mortiers anciens apportera plus de clarté au sujet de la connaissance empirique des propriétés de la chaux pour certaines applications ou durant certaines époques de l'histoire.

\section{Remerciements}

L'étude s'inscrit dans le cadre du projet « Pôle d'Attraction Interuniversitaire - PAI V/P9 ». Nous voulons remercier le «Service Public Fédéral de Programmation Politique Scientifique » de Belgique qui s'occupe du financement de ce projet. Le premier auteur travaille actuellement comme Aspirant du Fonds de la Recherche Scientifique - Flandre (FWO - Vlaanderen), qui est remercié pour son support financier. Anne Brutsaert et Marianne Deckers sont remerciées pour le prélèvement soigneux des échantillons.

\section{Bibliographie}

Boynton, R. S., 1966. Chemistry and technology of lime and limestone, New York, John Wiley \& Sons.
Brulet, R. et Verslype, L., 2001. Naissance et évolution de la ville : analyse du bâti antique à Tournai, in M. Lodewijckx (éd.), Album amicorum Joseph Remi Mertens. Acta archaeologica Lovaniensia. Monographiae, 13, p. 101-109.

Callebaut, K., Elsen, J., Van Balen, K. et Viaene, W., 2001. Nineteenth century hydraulic restauration mortars in the Saint Michael's Church (Leuven, Belgium) Natural hydraulic lime or cement?, Cement and Concrete Research, 31, p. 397-403.

Camerman, C., 1944. La pierre de Tournai : Son gisement, sa structure et ses propriétés, son emploi actuel, Mémoires de la Société belge de Géologie, de Paléontologie et d'Hydrogéologie, Nouvelle série, in $4^{\circ}$, p. 1.

Coutelas, A., Godard, G., Blanc, Ph. et Person, A., 2004. Les mortiers hydrauliques : synthèse bibliographique et premiers résultats sur des mortiers de Gaulle romaine, Revue d'Archéométrie, 28, p. 127-139.

Elsen, J., Brutsaert, A., Deckers, M. et Brulet, R., 2004. Microscopical study of ancient mortars from Tournai (Belgium), Materials Characterization, 53, p. 289-294.

Gaillard, C., Hennebert, M. et Olivero, D., 1999. Lower Carboniferous Zoophycus from the Tournai area (Belgium), environmental and ethologic significance, Geobios, 32, (4), p. 513-524.

Hennebert, M. et Doremus, P., 1997. Carte géologique de Wallonie : Notice explicative, Antoing-Leuze 37/7-8, Ministère de la région walonne - Direction générale des ressources naturelles et de l'environnement, Belgique.

Ito, S., Nishikawa, T., Ito, Y. et Suzuki, K., 1992. Thermal behaviour of synthesized C-S-H, Proceedings of the $9^{\text {th }}$ International Congress on the Chemistry of Cement, Volume 1, New Delhi, India, p. 151-156.

Mertens, G., Elsen, J., Brutsaert, A., Deckers, M. et Brulet, R., 2005. Physico-chemical evolution of lime mortars from Tournai (Belgium), Proceedings of the International Building Lime Symposium 2005, Orlando, United States, p. 1-13.

Middendorf, B., Baronio, G., Callebaut, K. et Hughes, J. J., 2000. Chemical-mineralogical and physical-mechanical investigations of old mortars, Proceedings of the International RILEM-workshop "Historic mortars : characteristics and tests", Paisley, Scotland, p. 53-61.

Mortelmans, G., 1969. L'étage Tournaisien dans sa localité-type, Comptes rendus, $\sigma^{e}$ Congrès International de Stratigraphie et de Géologie du Carbonifere, Volume 1, Sheffield, p. 19-44.

Philippo, S., 1992. Étude pétrographique et géochimique du calcaire carbonifere du Tournaisis et sa relation avec certains problèmes de durabilité des bétons, Mémoire de Licence, Université de Louvain-la-Neuve, Belgique.

TaYlor, H. F. W., 1990. Cement Chemistry, Academic press, New York.

ArcheoSciences, revue d'archéométrie, 30, 2006, p. 61-65 